BMJ Open

Diabetes

Research

\& Care

\section{Improving performance in the detection and management of cystic fibrosis- related diabetes in the Mountain West Cystic Fibrosis Consortium}

To cite: Liou TG, Jensen JL, Allen SE, et al. Improving performance in the detection and management of cystic fibrosis-related diabetes in the Mountain West Cystic Fibrosis Consortium. BMJ Open Diabetes Research and Care 2016;4:e000183. doi:10.1136/bmjdrc-2015000183

Received 16 December 2015 Revised 10 March 2016 Accepted 2 April 2016

CrossMark

For numbered affiliations see end of article.

Correspondence to Dr Theodore G Liou; ted.liou@utah.edu

\section{ABSTRACT}

Objective: Cystic fibrosis (CF)-related diabetes (CFRD) is associated with increased morbidity and mortality. Improved detection and management may improve outcomes; however, actual practice falls short of published guidelines. We studied efforts to improve CFRD screening and management in the Mountain West CF Consortium (MWCFC).

Research design and methods: This is a prospective observational cohort study evaluating quality improvement by accredited CF centers in Arizona, Colorado, New Mexico, and Utah performed between 2002 and 2008. After Institutional Review Board (IRB) approval, centers evaluated adherence with CF Foundation guidelines for CFRD. Each center developed and implemented quality improvement plans to improve both screening and management. Centers were reassessed 1 year later.

Results: Initially, each CF center had low adherence with screening recommendations $(26.5 \%$ of eligible patients) that did not improve during the study. However, patients with confirmed CFRD markedly increased (141 (12\% of MWCFC patients) to 224 $(17 \%), p<0.001)$, and with improved adherence to management guidelines, patients with CFRD had increased weight (56.8-58.9 kg, $p<0.001)$, body mass index (21.1-21.4, $p=0.003)$, and weight-for-age $z-$ score $(-1.42$ to $-0.84, p<0.001)$. Quality improvement methods were specific to the practice settings of each center but shared the common goal of adhering to CFRD care guidelines. 1 year after implementation, no center significantly differed from any other in level of adherence to guidelines.

Conclusions: Improving adherence with CFRD care guidelines requires substantial effort and may be incompletely successful, particularly for CFRD screening, but the effort may significantly improve patient monitoring and clinically relevant outcomes such as weight.

Cystic fibrosis $(\mathrm{CF})$ is the most common genetically determined disease among those of northern European descent in the USA.

\section{Key messages}

Adherence to cystic fibrosis-related diabetes guidelines requires improvement.

- Quality improvement efforts tailored to specific performance sites differ in details but offer the best chance of successful implementation.

- Improved adherence produces objective improvement in outcomes that are clinically relevant to patients with cystic fibrosis.

Protein malabsorption, progressive lung disease, and early death characterize the disease, ${ }^{12}$ but CF-related diabetes (CFRD) is an increasingly common complication with substantial morbidity and negative survival effects. $^{3-6}$

Approximately $15 \%$ of patients with $\mathrm{CF}$ are affected by CFRD, often developing the disease in adolescence or young adulthood. ${ }^{347}$ CFRD is distinct from type I and type II diabetes. ${ }^{4}$ Patients have relative insulin deficiency but no immunological markers typical of type I diabetes, and ketoacidosis is rare. Compared with type II diabetes, obesity is infrequent, but some degree of insulin resistance is common. CFRD is associated with female gender, pancreatic insufficiency, worsened lung disease, more frequent pulmonary exacerbations, poorer nutritional status, and decreased survival. ${ }^{56}$

Because of the large clinical impact, the US CF Foundation, in association with the American Diabetes Association, maintains guidelines for the diagnosis and care of patients with CFRD, most recently updated in $2010 .^{8}$ Implementation of these guidelines was uneven across the more than 110 accredited $\mathrm{CF}$ care centers in the USA at the time of initiation of our quality improvement (QI) project and remains uneven despite years of effort. ${ }^{9} 10$ 
Recognizing the need to improve guideline adherence by $\mathrm{CF}$ care centers, we performed a prospective, observational, QI cohort study with two major goals. First, we sought to measure and improve the completeness of recommended screening of patients with CF for CFRD among participating centers. Second, we sought to measure and improve the level of adherence by CF care centers with guidelines for the care of patients with CFRD.

\section{RESEARCH DESIGN AND METHODS \\ Trial participation}

The personnel at accredited $\mathrm{CF}$ care centers in the Mountain West CF Consortium (MWCFC) agreed to participate in the trial. All trial procedures were reviewed and approved by the Investigational Review Board (IRB) at the host institution of each participating center and were also reviewed by the CF Foundation. The institutions included the University of Arizona, Tucson, Arizona, USA; Phoenix Children's Hospital, Phoenix, Arizona, USA; National Jewish Health, Denver, Colorado, USA; Children's Hospital Colorado, Aurora, Colorado, USA; the University of New Mexico, Albuquerque, New Mexico, USA; and the University of Utah, Salt Lake City, Utah, USA.

Each center developed plans to increase screening for CFRD and to improve monitoring and management of patients already diagnosed with CFRD. Every center tailored plans for each specific practice environment and agreed to external surveillance. ${ }^{11}$ Investigators from the University of Utah visited each center and performed audits of CFRD screening and monitoring prior to implementation of local QI plans. One full year after implementation, the audits were repeated.

Informed consent was not required to assess the completeness of screening for CFRD at participating centers. However, informed consent was required for patients with CFRD who were included in the assessments of adherence with CFRD guidelines by each participating center and for whom we collected patient-specific data. Informed consent was obtained from all patients of majority age. Assent and informed consent were obtained from patients with CF of minority age and their parents, respectively. Patient recruitment was initiated in January of 2003 and completed by April of 2005. Follow-up for the study was completed in 2007, and the data set was finalized in early 2008.

\section{CF Foundation Patient Registry Data}

To provide a background for interpretation of MWCFC results, we obtained permission from the CF Foundation to access CF Foundation Patient Registry (CFFPR) data to examine screening efforts for accredited centers in the USA. The data allow comparison between MWCFC centers participating in the study with all other accredited centers for the time period of the study.

Data collection for the CFFPR is approved annually by the IRB at each participating center. Written informed consent is obtained from each patient or guardian for each year of participation, and data obtained may be deleted both prospectively and retrospectively by patient request. Our use of CFFPR data was reviewed by the IRB at the University of Utah and was granted a waiver for additional informed consent.

\section{Data collection}

Research coordinators at each center identified all patients for whom screening for CFRD had been performed in 2002 in adherence with CF care guidelines. ${ }^{8}$ To derive a screening rate for each institution, the coordinators identified all patients followed at each institution in 2002. A repeat assessment of screening for CFRD in 2004 was performed for comparison of screening rates at each center before and after QI efforts.

To assess adherence with CFRD guidelines concerning care of individual patients with CFRD, we recruited patients 6 years and older at the participating centers that met one of four diagnostic criteria: (1) a documented oral glucose tolerance test (OGTT) result at $2 \mathrm{~h}$ after oral glucose load $\geq 200 \mathrm{mg} / \mathrm{dL}$, or (2) a fasting blood glucose (FBG) level $>126 \mathrm{mg} / \mathrm{dL}$ on two or more occasions, or (3) a FBG level $>126 \mathrm{mg} / \mathrm{dL}$ on one occasion with a random glucose level of $200 \mathrm{mg} / \mathrm{dL}$ or more, or (4) random glucose levels of $200 \mathrm{mg} / \mathrm{dL}$ or more on two or more occasions with symptoms attributable to hyperglycemia. Patients that were unable or unwilling to give consent, unwilling to undergo study procedures, already enrolled in another investigational study, pregnant, prisoners, or prior organ transplant recipients were excluded. Patients were censored for the second chart review if information was unavailable due to loss to follow-up, organ transplantation, or death prior to the end of the second review period.

We created a data collection tool using Access (Microsoft Corp, Bellevue, Washington, USA) to record data about patients with CFRD within each center pertinent to measuring outcomes and adherence of centers with CFRD care guidelines. A single investigator visited all sites in 2003 to review patient charts and collect data reflective of CFRD management. Patient information was collected on age, gender, insulin usage, height, weight, glycated hemoglobin (HbAlc), forced expiratory volume in $1 \mathrm{~s}\left(\mathrm{FEV}_{1}\right)$, ethnicity and race, number of clinic visits, hospitalizations, and number of acute exacerbations. $\mathrm{FEV}_{1}$ was normalized to percent of predicted $\mathrm{FEV}_{1}\left(\mathrm{FEV}_{1} \%\right)$ using National Health and Nutritional Evaluation Survey III equations. ${ }^{12}$ Information was gathered to determine whether the physical examinations and laboratory testing suggested by CF Foundation CFRD guidelines were performed on patients. These procedures included blood pressure, weight, height, retinal and foot examinations, and testing for HbAlc, lung function, sputum cultures, fasting hyperglycemia, urinalyses, and lipid profiles. Within 6 months of completion of the first round of chart reviews, each center devised a QI plan partially 
based on center-specific results and implemented a center-specific plan for improving adherence (tables 1 and 2). One year after each plan implementation, an investigator traveled to each site and performed a second chart review.
To enable comparison of screening for CFRD nationally, we classified patients in the CFFPR during 2002 and 2004 as receiving care primarily or not at a MWCFC care center participating in the study. Patients were excluded for prior lung transplantation, and they were censored for loss to

Table 1 QI plans by CF center for CFRD screening

Programme

National Jewish Health (Adult)

Phoenix Children's Hospital (Pediatric)

University of Arizona (combined Adult and Pediatric)

University of Utah (Primary Children's Medical Center, Pediatric)

University of Utah (Adult)

University of New Mexico (combined Pediatric and Adult)

\section{Plan elements}

1. Random glucose was included in routine laboratory obtained annually on all patients (at the clinic visit closest to their birthdays).

2. During weekly team meetings, the team reviewed all charts for patients scheduled for clinic that week. OGTT was then discussed with all patients over 10 years old who had not completed one in the prior year.

3. During team meetings in November, the team also reviewed and contacted all patients over 10 years old who had not completed an OGTT during the preceding year.

1. The team reviewed all patient charts the day prior to visits to identify patients unscreened by random glucose.

2. The team notified the providers who would offer random glucose and OGTT screening to all non-diabetes patients.

1. OGTT was added to annual laboratories in patients 10 years of age and older.

2. The team reviewed all patient charts prior to visits for annual laboratories to identify patients unscreened by random glucose during the prior year and also identified patients with poor weight gain or loss of lung function as needing CFRD screening.

3. The CF team notified the providers which patients needed screening. Providers would offer random glucose and OGTT screening to those and all non-diabetes patients that were older than 10 years of age.

4. Patients that needed screening were notified by mail prior to clinic to obtain an OGTT at an outside laboratory. If it was not performed by the clinic visit, patients were asked again to obtain the OGTT at an outside laboratory.

5. The staff revised the patient assessment form for clinic visits to flag the need for CFRD screening.

6. Patients would undergo fasting and $2 \mathrm{~h}$ postprandial glucose checks during the first $48 \mathrm{~h}$ of any hospital admission.

1. All adolescent and adult patients admitted for pulmonary exacerbations were screened with premeal, bedtime and 0200 finger-stick glucose measurements for the first $48 \mathrm{~h}$ of hospitalization to detect illness-associated impaired glucose tolerance to prompt additional screening later.

1. OGTT was added to the usual tests and procedures during annual visits.

2. The nurse coordinator or dietician notified families to come to clinic with the patient fasting as part of clinic preparations the week prior to the visit.

3. The clinic tried to schedule visits with fasting OGTT in the morning, preferably as a first appointment.

4. OGTT was rescheduled whenever planned but not performed.

1. OGTT was planned for posthospitalization visits to maximize the chance that patients were stable at the time of testing.

2. The nurse coordinator identified patients without CFRD or OGTT within a year of a clinic visit during clinic prep and called to ask each patient to come to clinic fasting.

3. When not performed at clinic, the physician and staff gave patients prescriptions to complete the test either at the clinic on another day or at a facility convenient to the patient.

1. OGTT was added as a test for annual visits in clinic.

2. The CF dietician kept track of patients in need of OGTT and made sure to tell patients prior to the clinic date to come fasting.

3. OGTT was limited to approximately $3 /$ week to facilitate first morning appointments. 


\section{Table 2 QI plans by CF center to improve CFRD care}

Programme
Children's Hospital Colorado (Pediatric)

National Jewish Health (Adult)

Phoenix Children's Hospital (Pediatric)

University of Arizona (combined Adult and Pediatric)

University of Utah (Primary Children's Medical Center, Pediatric)

University of Utah (Adult)

University of New Mexico (combined Pediatric and Adult)

\section{Plan elements}

1. All patients with an abnormal OGTT were referred to the Barbara Davis Diabetes Center. Routine diabetes care was then coordinated by that clinic.

2. The CF dietician met with all patients to help coordinate care with the Barbara Davis Center dietician.

1. At time of diagnosis of CFRD and for all patients with previously diagnosed CFRD, clinic staff offered urine microalbumin-to-creatinine ratio testing and recommended an annual dilated retinal examination.

2. Clinic staff offered insulin therapy to patients with diagnosed CFRD and recorded the decision to initiate the therapy.

3. Staff recorded the number of acute pulmonary exacerbations, pulmonary function tests and measurements of BMI for the year before and the year after a diagnosis of CFRD.

1. The dietician or other personnel would track that patients were getting tests and referrals. At preclinic team meetings, the staff notified physician providers which patients needed CFRD follow-up care.

2. The staff revised the patient assessment form for clinic visits to flag the need for CFRD-focused physical examinations and referrals and provided a check box to indicate that tests were actually performed in the prior year.

3. Laboratory testing was ordered prior to clinic visits, and patients were asked to obtain testing prior to clinic. At clinic, patients were reminded to obtain the tests if they had not been performed.

4. The team generally increased the discussion and education about CFRD with patients at every visit and explained the need for testing. Patients that failed to get tests were provided additional education.

1. Physicians were reminded by clinic staff to perform appropriate CFRD-related physical examinations.

2. Patients were referred annually for dilated eye examinations.

3. Physicians were reminded by clinic staff to include urinary microalbumin and serum fructosamine in annual laboratories.

1. Dietician counted the procedures performed for CFRD follow-up and informed the clinicians.

2. CFRD follow-up measures were included as part of the annual review by the clinical nurse coordinator for each patient.

3. Input from the dietician and other personnel on CFRD follow-up was reported to the clinicians.

1. Clinic staff reminded the physician to perform appropriate CFRD-related physical examinations.

2. Patients were referred by clinic staff for dilated eye examinations. If not performed by the next visit, patients were reminded.

3. The local electronic medical record physical examination form was modified to highlight the need for CFRD-specific examinations such as foot examinations to cue the clinicians to perform these follow-up examinations.

4. Standard clinic laboratory orders were modified to automatically include $\mathrm{HbA1c}$ and urine microalbuminuria testing.

1. Clinic staff kept track of appropriate CFRD-related physical examinations and tests.

2. Staff provided education to each other and to patients to encourage increased numbers of CFRD-specific physical examination elements and to order CFRD-specific laboratory tests and referrals for retinal eye examinations.

BMI, body mass index; CF, cystic fibrosis; CFRD, CF-related diabetes; HbA1c, glycated hemoglobin; OGTT, oral glucose tolerance test; QI, quality improvement.

follow-up or new lung transplantation during the 20022004 period. Cross-matching of patients between MWCFC center data and CFFPR national study-derived data was not feasible due to lack of consent for patients enrolled in the MWCFC study or specific CF Foundation or institutional support for cross-matching by specific patient identifiers.

\section{Statistical analysis}

We use the statistical system $\mathrm{R}$ for all analyses. ${ }^{13}$ Comparison of parameters between first and second chart reviews to look for improvements associated with implementation of QI programmes used McNemar's paired $\mathrm{t}$ tests for continuous data. ${ }^{14}$ Comparisons 
including patients with complete data or with data in only the first or second year of study representing a more complete sampling of each participating center's patient population used Pearson's $\chi^{2}$ or Fisher's exact tests for categorical data and Welch two-sample $t$ tests for continuous data. ${ }^{14}$ These two sets of parameter comparisons allow us first to explore whether the QI effort was associated with improved CFRD outcomes and second to discern whether there was evidence that analysis of patients with 2 years of data rather than just 1 year might have been influenced by bias due to patient selection. Finding such bias would provide evidence that data were not missing at random.

Comparisons of screening by FBG alone or OGTT between MWCFC and all other accredited care centers using data from the CFFPR used generalized linear models (logistic regression). Univariate relationships were re-examined in multivariate models that corrected for clinical characteristics that might have influenced the likelihood of screening. These included frequency of clinic visits, $\mathrm{FEV}_{1} \%$, number of acute pulmonary exacerbations in the year prior to study participation, and 5-year predicted survival as an overall measure of disease status.

Missingness of measurements recommended by CFRD care guidelines were the outcomes used to assess adherence, thus no imputation was planned or performed. However, for data from patients with CFRD where the actual measurement was of interest, we performed multiple imputation with chained equations, ${ }^{15}$ examined individual imputed data sets, and repeated analyses looking for evidence that data were not missing completely at random (a more stringent level of assessment of missingness than described above), and thus may have introduced bias. For the imputation analyses, we included all patients with 2 years of data collection including both those with complete and incomplete records as well as patients with only 1 year of data, treating them as missing all the data from the other potential year of participation.

\section{RESULTS}

\section{Adherence with screening guidelines}

There were 1163 patients in 2002 and 1316 patients in 2004 followed at the two adult, two pediatric, and three combined-age participating CF centers in the MWCFC. Nearly all patients (91\%) underwent some type of screening in 2002 (by random blood glucose, FBG alone, or OGTT), but there was a significant decrease to $75 \%$ by 2004 . The rate of screening using the more stringent recommendation in CFRD guidelines, FBG alone or OGTT, was substantially lower and did not change between 2002 and 2004 (table 3).

\section{Patients with CFRD}

There were 141 patients in 2002 (12.1\%) and 224 patients in 2004 (17.0\%) diagnosed with CFRD (table 3 ), a statistically significant increase in prevalence $\left(\chi^{2}, p<0.001\right)$. Eight patients were excluded because their diagnoses of CFRD were reversed; two patients were too young for the study; three patients underwent transplantation prior to the first chart review. Three patients were excluded from the second chart review due to new lung transplants during the study; three additional patients were excluded due to incarceration, death, and loss to follow-up. After exclusions, a total of 92 patients consented to participate in either the first (5 patients) or second (23) or both chart reviews (64).

\section{CFRD chart reviews for adherence with care guidelines}

Data collected (table 4) showed that the population of patients studied in each year of study was comparable to the general population of patients with CF in the USA. ${ }^{9}$ Height was increased due to expected growth in the pediatric patients. Weight and body mass index (BMI) increased (figure 1), and there was an accompanying increase in weight-for-age z-score; the increases in weight measures were statistically significant and included patients for whom no growth in stature was expected or occurred. Finally, $\mathrm{FEV}_{1} \%$ dropped significantly between chart reviews for patients participating in both years of data collection.

There was no change in the numbers of patients with fasting hyperglycemia or the numbers of patients using insulin (table 5). There was a significant decrease in the

Table 3 Screening for CFRD

\begin{tabular}{|c|c|c|c|}
\hline Patients & 2002 & 2004 & $p$ Value \\
\hline MWCFC total & 1163 & 1316 & - \\
\hline $\begin{array}{l}\text { Eligible for OGTT } \\
\text { (no prior CFRD diagnosis) }\end{array}$ & 1022 & 1092 & - \\
\hline Tested by OGTT & 162 & 132 & $0.08^{\star}$ \\
\hline Tested by FBG alone & 109 & 149 & $0.12^{*}$ \\
\hline $\begin{array}{l}\text { Screened at least once for } \\
\text { CFRD by any method } \\
\text { (\% of total) }\end{array}$ & $1054(91)$ & $982(75)$ & $<0.001^{\star}$ \\
\hline $\begin{array}{l}\text { Confirmed with CFRD } \\
\text { (\% of total) }\end{array}$ & $141(12)$ & $224(17)$ & $<0.001 \dagger$ \\
\hline
\end{tabular}

${ }^{*}$ Despite specific interventions to improve, screening procedures for CFRD failed to improve during the study. OGTT numbers and FBG measurements were unchanged while random blood glucose measurements were significantly reduced. Considering the more sensitive and specific combination of FBG alone or OGTT shows that $26.5 \%$ of all eligible patients in 2002 and $25.7 \%$ in 2005 were screened for CFRD in MWCFC study centers. The change in testing for this combination was not significantly improved. These observations cannot be explained by the increased numbers of patients included in the second chart review in the study. †Although testing for CFRD decreased during the study, the number of patients with a diagnosis of CFRD significantly increased, a change not explained by the increase in overall population of patients with CF. Nationally, all CF centers had a decrease in screening using random blood glucose, FBG alone or OGTT from $80.5 \%$ in 2002 to $73.3 \%$ in 2004 , a change that parallels the decrease in MWCFC centers. ${ }^{9} 16$

CF, cystic fibrosis; CFRD, CF-related diabetes; FBG, fasting blood glucose; MWCFC, Mountain West CF Consortium; OGTT, oral glucose tolerance test. 
Table 4 Characteristics and outcomes of patients with CFRD after QI efforts

\begin{tabular}{|c|c|c|c|}
\hline & $\begin{array}{l}\begin{array}{l}\text { First chart } \\
\text { review } n=69\end{array}\end{array}$ & $\begin{array}{l}\text { Second chart } \\
\text { review } n=87\end{array}$ & p Value \\
\hline Age in & $26.1(11.7)$ & $25.1(11.1)$ & 0.56 \\
\hline Male, & & & 92 \\
\hline , n (\%) & )) & 6) & 0.89 \\
\hline (SD) & 163.41 & 170.73 & $0.012 \dagger$ \\
\hline $\begin{array}{l}\text { Height-for-age } \\
\text { z-score (SD) }\end{array}$ & 0148 & 126 & $0.26 \S$ \\
\hline (SD) & & & 001 . \\
\hline & 1) & - & $0.003 \dagger$ \\
\hline $\begin{array}{l}\text { Weight-for-age } \\
\text { z-score (SD)ף }\end{array}$ & $-1.43(0.76)$ & $-0.84(0.75)$ & $<0.001 \dagger$ \\
\hline $\mathrm{FEV}_{1} \%(\mathrm{SD})^{\star \star}$ & $65.6(25.5)$ & $61.9(24.0)$ & $0.008^{\dagger}$ \\
\hline
\end{tabular}

*Weight change reported is uncorrected for age or growth. †McNemar's paired t test. There were 63 patients with height measurement, 62 patients with weight, 62 patients with BMI, and 54 patients with $\mathrm{FEV}_{1} \%$ data from both chart reviews available for paired $t$ tests. Significant changes in weight, weight-for-age z-score, $\mathrm{BMI}$, and $\mathrm{FEV}_{1} \%$ persist when only adults are included or only patients without growth in height are included.

fHeight-for-age $z$-score is calculated using the method of Dibley et $a l^{17}$ and Lai et al, ${ }^{18}$ as we have performed previously for weight-for-age z-score. ${ }^{6}$

\$When tested using $t$ test, $p=0.26$, indicating that the height characteristics of the average patient followed at the study centers did not change during the study period. However, when testing patients participating in both years of the study, McNemar's paired $t$ test detects that our group of study patients included adolescents who grew in stature during the study.

qWeight-for-age z-score calculated as we have performed previously. ${ }^{6}$

${ }^{\star *}$ Annual maximum recorded value. The maximum value is chosen because it is the most reliably reproducible measurement. ${ }^{6}$ BMI, body mass index; CFRD, cystic fibrosis-related diabetes; $\mathrm{FEV}_{1} \%$, percent of predicted forced expiratory volume in $1 \mathrm{~s}$; QI, quality improvement.

number of patients visiting clinic six or more times in a year. There were significant increases in the numbers of retinal eye examinations and foot examinations performed. The number of urinalyses obtained from patients with CFRD was unchanged, but the frequency of inclusion of testing for albuminuria was markedly improved. Similarly, there was no change in the number of patients undergoing HbAlc tests during the year nor was there a great increase in the number of patients undergoing testing quarterly, but the number of $\mathrm{HbAlc}$ measurements per patient increased markedly.

\section{Comparison of the MWCFC to other US CF centers}

Screening rates for CFRD using FBG alone or OGTT were low throughout US accredited CF centers in 2002 but significantly increased through 2004 by $41 \%$ for non-MWCFC centers and $71 \%$ for centers participating in the MWCFC QI project (table 6). The odds of being screened for CFRD were significantly higher at MWCFC centers compared with all other US CF centers by about $80 \%$ in 2002 and 2004. In multivariate analyses, corrections for number of clinic visits, best $\mathrm{FEV}_{1} \%$ in each study year, number of prior acute pulmonary exacerbations, and 5-year predicted survival ${ }^{6}$ did not significantly change these results.

\section{Missingness of data}

Out of 92 patients enrolled in the overall study, 64 participated in both chart reviews. We examined the potential bias due to missingness of data that was not missing at random or not missing completely at random on the evaluation of effects associated with implementation of QI programmes. We considered both the 64 patients with participation throughout the study who had sporadically missing individual pieces of data as well as the 28 patients that were missing all data from either the first or second year of the study.

Comparison of aggregated data using unpaired tests between 2002 and 2004 showed no qualitative difference from paired $t$ tests for height, weight, BMI, weight-for-age $\mathrm{z}$-score, or HbAlc testing. However, unpaired t test showed no difference in $\mathrm{FEV}_{1} \%$ between the two study years while paired testing revealed a strong statistically significant difference. This indicated that lung disease progressed for individual patients, although the overall level of disease in each center remained unchanged. Overall, these tests suggest that selection of patients with 2 years of data did not introduce a bias related to missing data that was not missing at random. However, the findings with $\mathrm{FEV}_{1} \%$ values suggested further evaluation of missingness to consider whether there was evidence that data were not missing completely at random.

Multiple imputation with chained equations ${ }^{15}$ with 50 iterations using predictive mean matching produced plausible distributions of values for missing data on inspection of density plots comparing imputed to nonmissing data but suggested that missing $\mathrm{FEV}_{1} \%$ values in the second year of chart review would have been somewhat worse than non-missing measurements. $\mathrm{FEV}_{1} \%$ values had density distributions in 10 imputed data sets that were somewhat lower than the density distribution of actual data. However, there was no significant or clinically relevant shift in median or mean values. Repetition of statistical testing for changes in height, weight, BMI, and $\mathrm{FEV}_{1} \%$ with imputed data sets between the two study years showed no evidence that data were not missing completely at random or that final results were significantly changed by missingness.

\section{DISCUSSION}

CFRD is a common complication of CF itself and requires specific care as outlined in the $\mathrm{CF}$ Foundation Care Guidelines. ${ }^{8}$ Our efforts to improve screening for this condition and to improve the quality of care given to patients bearing the diagnosis had mixed, although generally positive results.

The number of confirmed diagnoses increased significantly during the study. However, the increase unfortunately was not clearly due to increased frequency of screening, a major goal of the QI project (table 3). As 

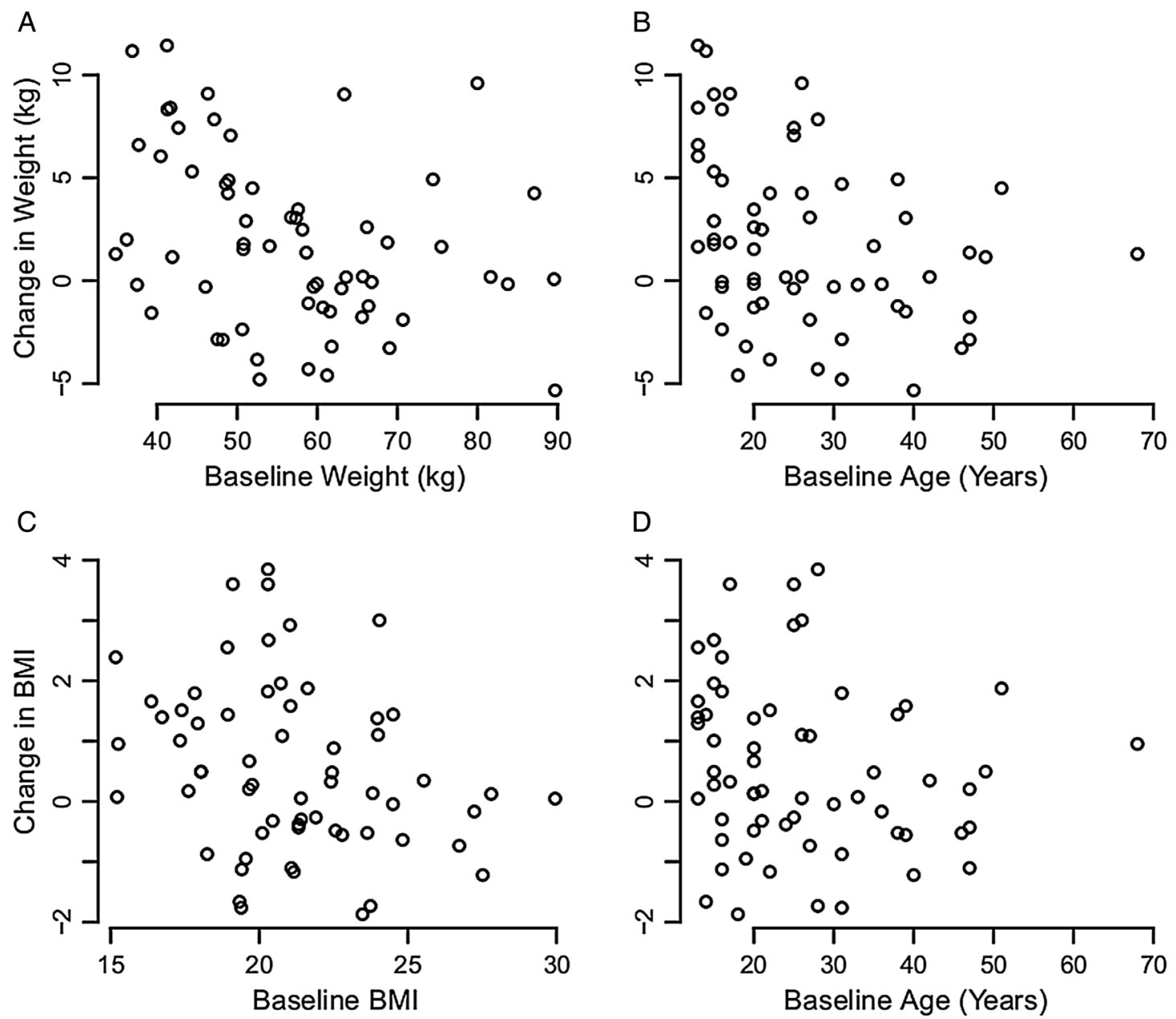

Figure 1 Changes in weight and body mass index (BMI) associated with quality improvement. (A and $B)$ Show change in weight relative to beginning weight and age. Most patients have an increase in weight during the study period which is not due to growth. ( $C$ and $D)$ Show similar improvements in BMI during the study period. The improvements were independent of cystic fibrosis center identities.

part of study development, all clinic practitioners received additional education on the clinical importance and characteristics of CFRD. The increased number of confirmed diagnoses may indicate that practitioners focused their screening efforts on patients for whom they had increased clinical suspicion of CFRD, that were not included in the study, or perhaps that were diagnosed during the 6-month period when QI plans were being formulated at each center: outcomes that we did not measure.

There were three distinct methods for screening: OGTT, a random blood sugar measurement following an abnormal FBG, and two abnormal random blood sugar measurements. Unfortunately, no test used for CFRD screening among study participants was increased in application at the time of the second chart review compared with the first chart review, highlighting the difficulty of obtaining these tests (table 3).

OGTT is mildly uncomfortable due to fasting and repeated blood draws and may be technically difficult to obtain during an already busy multidisciplinary clinic visit. Obtaining fasting blood is complicated by patients that arrive in clinic without fasting and later fail to obtain testing on non-clinic visit days because of the inconvenience of fasting and the need for a timed blood draw. These issues were the most consistently reported barrier to improving the rate of CFRD screening among the MWCFC centers (table 7).

Confirmation of CFRD by random blood glucose measurement after an abnormal FBG is slightly easier to perform because timing of the random blood glucose is not so stringent, but still requires fasting and two blood draws. This screening strategy may also miss the substantial number of patients with CFRD without fasting hyperglycemia. ${ }^{4} 10$

Screening by obtaining two random, high, blood glucose measurements avoids asking a patient to fast prior to a clinic visit, but it requires careful tracking of random blood glucoses over time to identify two abnormal values consistent with CFRD. The unscheduled nature of such testing introduces the challenge of tracking multiple serial results with varying intervals in order to make a diagnosis. This is further complicated by the possibility of false-positive results for patients with an 
Table 5 Measures reflective of center adherence with CFRD care guidelines

\begin{tabular}{|c|c|c|c|}
\hline $\begin{array}{l}\text { Recommended test, } \\
\text { results are } \mathrm{n} \text { and per } \\
\text { cent of reviewed } \\
\text { patients }\end{array}$ & $\begin{array}{l}\text { First } \\
\text { chart } \\
\text { review } \\
\mathrm{n}=69\end{array}$ & $\begin{array}{l}\text { Second } \\
\text { chart } \\
\text { review } \\
\mathrm{n}=87 \\
\end{array}$ & p Value \\
\hline & )) & 7 & 0.00 \\
\hline $\begin{array}{l}\text { Patients with more } \\
\text { than } 6 \text { clinic visits } \\
\text { annually } \dagger\end{array}$ & & & $0.002^{*}$ \\
\hline $\begin{array}{l}\text { eye } \\
\text { lation }\end{array}$ & $14(20)$ & 38 & $0.004^{*}$ \\
\hline nations & $14(20)$ & 4) & $0.004^{*}$ \\
\hline inuria testing & & & $<0.001^{*}$ \\
\hline $\begin{array}{l}\text { Any HbA1c } \\
\text { measurements }\end{array}$ & 52 & 69 & $0.69^{\star}$ \\
\hline $\begin{array}{l}3 \text { or more } \mathrm{HbA} 1 \mathrm{c} \\
\text { measurements in } \\
1 \text { year, } \mathrm{n}(\%)\end{array}$ & $1(1)$ & 6) & $0.005^{\star}$ \\
\hline \multicolumn{4}{|c|}{$\begin{array}{l}\text { *Pearson's } \chi^{2} \text {. } \\
\text { †The CF Foundation recommends four visits annually for routine } \\
\text { follow-up and maintenance of health. Additional visits are } \\
\text { generally for acute illnesses, and a reduction in these visits is a } \\
\text { generally desirable outcome. } \\
\text { CF, cystic fibrosis; CFRD, CF-related diabetes; HbA1c, glycated } \\
\text { hemoglobin. }\end{array}$} \\
\hline
\end{tabular}

ongoing acute illness. Those patients may be subject to a disease-specific bias that favors finding false-positive results for frequently hospitalized patients. Frequent sick visits and hospitalizations may increase the number of random blood sugar measurements at times when increased glucose values are much less specific for diagnosing CFRD.

Complementary data regarding screening efforts for CFRD from the CFFPR allow comparison between MWCFC and all other accredited US care centers (table 6). Owing to consenting and enrolment differences, these data overlap but are not directly comparable to data recorded among participants in our QI study. MWCFC screening results using FBG alone or OGTT were better than all others, a relationship that persisted in lesser degree through 2011 (the last year of CFFPR data currently available to our study group) even as all centers in the USA had marked improvements in screening rates. However, the results for all centers continued to fall short of complete adherence to CFRD screening guidelines through $2011 .^{10}$

In contrast to modest recorded results from trying to increase frequency of screening efforts to establish a diagnosis, we had marked improvements in management of patients with confirmed CFRD. The number of clinic visits was reduced for these patients, indirectly indicating a decrease in encounters for acute illness, even while we increased the numbers of examinations specific for CFRD follow-up. The increased attention to CFRD management was associated with a significant improvement in weight, BMI, and weight-for-age z-score that persisted even when we corrected for the effects of normal growth in height among the children expected to grow or focused exclusively on adults (figure 1). Unfortunately, a significant drop in lung function was also observed during the study period (table 4). This drop, however, was not greater than observed in the national CFFPR for the period of study. ${ }^{9} 16$

The evaluation of adherence to suggested CFRD care guidelines was hampered by the widely varying settings and styles of practice found within the MWCFC. However, this problem reflects the real-world difficulties that likely exist in adapting a single set of guidelines to over 110 care centers. Since this was a non-randomized and non-blinded trial that was observational for patients but involved a change in practice at the provider level, we expected that there would be an observer effect reflected in improvements in adherence to guidelines for making the diagnosis of CFRD. That we found no

Table 6 Screening comparison between MWCFC and all other US CF centers

\begin{tabular}{|c|c|c|c|c|}
\hline & \multicolumn{2}{|c|}{ Eligible patients screened for CFRD } & \multirow[b]{2}{*}{ OR $^{*}$} & \multirow[b]{2}{*}{$95 \% \mathrm{Cl}^{*}$} \\
\hline & National (n) screening rate & MWCFC (n) screening rate & & \\
\hline 02 & 10 & 120 & & \\
\hline & & & & \\
\hline OR & & 1.7 & & \\
\hline $95 \% \mathrm{Cl}^{*}$ & 1.38 to 1.45 & 1.57 to 1.85 & & \\
\hline \multicolumn{5}{|c|}{$\begin{array}{l}\text { *ORs calculated using generalized linear models (logistic regression) with screening by FBG alone or OGTT as the dependent variable. The } \\
95 \% \text { Cls all indicate a significance level of } p<0.001 \text {. } \\
\dagger \text { The n's and screenings reported for MWCFC care centers for } 2002 \text { and } 2004 \text { differ from those reported in table } 1 \text {. More patients were } \\
\text { screened and fewer were eligible for the study compared with patients included in the CFFPR. The differences are due to the exemption from } \\
\text { consent for measuring center adherence to screening guidelines within the QI study compared with required consent for inclusion in the } \\
\text { CFFPR, differences in determination of primary care center (and thus study participation eligibility) by the local centers versus the CFFPR and } \\
\text { the option afforded to CFFPR patients to withdraw consent with retroactive data erasure at any time. Differences in patients included in the } \\
\text { CFFPR compared with the MWCFC study account for the statistically significant improvement in CFRD screening that was not captured in the } \\
\text { current study. } \\
\text { fBy } 2011 \text {, screening rates utilizing either FBG alone or OGTT had risen to } 23 \% \text { nationally and } 29 \% \text { in the original MWCFC centers, } \\
\text { maintaining an OR of } 1.26 \text { (95\% Cl } 1.12 \text { to } 1.41, p<0.001 \text { ) for screening in the MWCFC compared with the rest of US centers. } \\
\text { CF, cystic fibrosis; CFRD, CF-related diabetes; CFFPR, CF Foundation Patient Registry; FBG, fasting blood glucose; MWCFC, Mountain } \\
\text { West CF Consortium; OGTT, oral glucose tolerance test; QI, quality improvement. }\end{array}$} \\
\hline
\end{tabular}


Table 7 Discovered barriers to quality improvement

Programme

National Jewish Health (Adult)

Phoenix Children's Hospital (Pediatric)

University of Arizona

(combined Adult and Pediatric)

University of Utah (Primary Children's

Medical Center, Pediatric)

University of Utah (Adult)

University of New Mexico

(combined Pediatric and Adult)

\section{Barriers}

1. Hospital policy required that a limited number of OGTT tests could be performed each day since the patient was required to stay in the laboratory throughout the time of the test. A new protocol was developed by the hospital laboratory to provide 2 regularly scheduled appointment slots for OGTTs on the days of the CF clinics.

2. Many patients lived a long distance from the clinic and wanted to avoid excessively long periods of fasting. A standard laboratory order form (including dose of glucose based on weight) was developed, so that patients could obtain an OGTT at outlying hospitals.

1. All clinics were in the afternoon which meant a long day of fasting or giving up a morning to do the OGTT and then spending the afternoon in CF clinic.

2. Patients greatly disliked the length of time it took to undergo OGTT.

1. There was no laboratory available at clinic at the beginning of the study period. All studies were obtained at outside laboratories, and some results were not reported back to the CF clinic. Outside laboratories did not always know how to dose glucola for children and increased side effects like vomiting and rebound hypoglycemia.

2. Timing of $2 \mathrm{~h}$ postglucola challenge glucose measurements were logistically difficult in clinic even after a laboratory facility was started in clinic. The frequency of quarterly $\mathrm{HbA} 1 \mathrm{c}$ measurements increased the logistical difficulties.

3. Some providers and patient families were uncomfortable with OGTT for younger patients.

4. All tracking was manual and difficult to perform.

5. There was no single endocrinologist identified to provide CFRD education increasing the potential for confusion about the disease and testing.

6. Patients and families did not like the extra cost of testing or the extra time.

7. Children often disliked the taste of glucola and had nausea and vomiting, and prediabetic patients would have insulin spikes followed by symptomatic hypoglycemia.

None reported.

1. Standardization of procedures was difficult.

2. The need for education of personnel that CFRD screening and monitoring are expectations for the entire clinic population.

3. Patients did not like the OGTT because of the time required to complete the testing.

4. Patients often failed to come fasting or to even come to clinic when an OGTT was anticipated.

5. Small children were unable to tolerate glucola, and modified testing was required to obtain results.

1. Patients frequently failed to come to clinic fasting or simply declined testing during clinic.

2. Patients infrequently returned to clinic for OGTT and rarely went to an outside facility to obtain the test.

1. Patients sometimes refused testing.

2. Patients failed to show for some clinic visits, and some patients limited visits to clinic to once per year.

CF, cystic fibrosis; CFRD, CF-related diabetes; HbA1c, glycated hemoglobin; OGTT, oral glucose tolerance test.

evidence of such bias underscores how difficult it may be to implement care guidelines even among caretakers motivated by the knowledge that they are being watched. However, we cannot exclude that the increase in diagnoses during the study may have been due to non-systematically study-associated increased attention for patients with clinical circumstances that raised the suspicion of CFRD and that participation in the study increased CFRD screening during the time allowed to each center to create QI plans.

It is possible that the improvements in measures of weight were spurious and not associated with increased efforts to improve CFRD management. However, observations beyond the end of the study suggest that these particular gains were sustained (not shown), and that efforts at improving management of patients with 
confirmed CFRD were more successful than for increasing screening of previously undiagnosed patients. Finally, our study was limited somewhat by missingness of data. However, evaluation of data from patients with 2 years of data versus patients with 1 or 2 years of data produced no indication that data were not missing at random, and multiple imputation by chained equations of missing data for height and weight produced no evidence of hidden clinically relevant biases due to missingness that was not completely at random. Imputation of $\mathrm{FEV}_{1} \%$ suggested that patients with missing data in the second year of the study might have had slightly lower values than for patients that had actual data; however, this potential bias had marginal statistical impact with no impact on interpretation of the change in $\mathrm{FEV}_{1} \%$ between the two chart reviews in the study.

CFRD is an increasingly common complication of CF. Comprehensive recommendations for screening and management have been disseminated by the $\mathrm{CF}$ Foundation, but we found those guidelines to be difficult to implement despite great motivation. The passage of additional time since our study has not resulted in sufficiently improved adherence by CF centers. ${ }^{10}$ Nevertheless, even with partial improvement and incomplete adherence, there were detectable improvements in clinical outcomes that imply better prognoses for individuals with CF, underscoring the worth of QI efforts.

\author{
Author affiliations \\ ${ }^{1}$ University of Utah, Salt Lake City, Utah, USA \\ ${ }^{2}$ University of New Mexico, Albuquerque, New Mexico, USA \\ ${ }^{3}$ National Jewish Health, Denver, Colorado, USA \\ ${ }^{4}$ University of Arizona, Tucson, Arizona, USA \\ USA \\ ${ }^{6}$ Phoenix Children's Hospital, Phoenix, Arizona, USA \\ ${ }^{7}$ Loma Linda University, Loma Linda, California, USA \\ ${ }^{8}$ Children's Hospital Colorado, Aurora, Colorado, USA \\ ${ }^{9}$ University of Colorado, Aurora, Colorado, USA \\ ${ }^{10}$ Cystic Fibrosis Foundation, Bethesda, Maryland, USA
}

${ }^{5}$ Colorado Department of Public Health and Environment, Denver, Colorado,

Acknowledgements TGL takes primary responsibility as the guarantor for the work and resulting manuscript, but all authors participated in study design, performance, and manuscript review and decision to publish. All authors have complete immediate access to the data. This project was funded by a Quality Improvement Grant from the US Cystic Fibrosis Foundation (Bethesda,

Maryland, USA), Liou01COQ1. TGL received additional support from a Harry Shwachman Clinical Investigator, Liou01A0, and Clinical Research, Liou 14P0 and Loiu 14A0, Awards from the CF Foundation, a Margolis Family Foundation of Utah award and NIH awards during the course of the study, R21HL084341, R01HL125520. The CF Foundation reviewed the manuscript for use of CFFPR data that maintains privacy of protected health information. A preliminary version of this work was presented in abstract and poster form at the 20th North American Cystic Fibrosis Conference, Denver, Colorado, USA, November, 2006.

Contributors TGL designed the study, performed analysis, led manuscript writing and helped maintain funding; JLJ assisted study design, enrolled patients, collected data, monitored multiple sites for data accuracy, assisted analysis, reviewed and edited the manuscript, managed funding and subcontracting with participating sites; JLJ shares first author credit for this project. SEA, SJB, MAB, BC, JK, CM, KP, PR, LMR, KO, and JSW managed IRB submissions at their respective participating sites, enrolled patients, collected data, reviewed and edited the manuscript; KAP assisted with IRB submission, enrolled patients, collected data, monitored some participating sites, assisted analysis, reviewed and edited the manuscript; DY assisted analysis, reviewed and edited the manuscript; BCM initiated the study idea, obtained funding, assisted study design, reviewed and edited the manuscript. The order of listing in the byline shows first authors, an alphabetized list of middle authors and the senior author.

Funding National Heart, Lung, and Blood Institute, R21HL084341, R01HL125520; Ben B and Iris M Margolis Family Foundation of Utah; Cystic Fibrosis Foundation, Liou01C0Q1, Liou01A0, Liou14P0, Liou14A0.

Disclaimer Opinions expressed in this work are solely those of the authors and do not necessarily express opinions or views of the CF Foundation, the Margolis Foundation, the NIH or the US Government.

Competing interests None declared.

Ethics approval Institutional Review Boards at each institution participating in this study.

Provenance and peer review Not commissioned; externally peer reviewed.

Data sharing statement Original data in de-identified form are available to qualified researchers by request to the corresponding author.

Open Access This is an Open Access article distributed in accordance with the Creative Commons Attribution Non Commercial (CC BY-NC 4.0) license, which permits others to distribute, remix, adapt, build upon this work noncommercially, and license their derivative works on different terms, provided the original work is properly cited and the use is non-commercial. See: http:// creativecommons.org/licenses/by-nc/4.0/

\section{REFERENCES}

1. Andersen DH. Cystic fibrosis of the pancreas and its relation to celiac disease: a clinical and pathologic study. Am J Child 1938;56:344-99.

2. Rowe SM, Miller S, Sorscher EJ. Cystic fibrosis. N Engl J Med 2005;352:1992-2001.

3. Lanng S, Thorsteinsson B, Lund-Andersen C, et al. Diabetes mellitus in Danish cystic fibrosis patients: prevalence and late diabetic complications. Acta Paediatr 1994;83:72-7.

4. Moran A, Doherty L, Wang X, et al. Abnormal glucose metabolism in cystic fibrosis. J Pediatr 1998;133:10-17.

5. Marshall BC, Butler SM, Stoddard M, et al. Epidemiology of cystic fibrosis-related diabetes. J Pediatr 2005;146:681-7.

6. Liou TG, Adler FR, Fitzsimmons SC, et al. Predictive 5-year survivorship model of cystic fibrosis. Am J Epidemiol 2001:153:345-52.

7. Moran A, Dunitz J, Nathan B, et al. Cystic fibrosis-related diabetes: current trends in prevalence, incidence, and mortality. Diabetes Care 2009;32:1626-31.

8. Moran A, Brunzell C, Cohen RC, et al. Clinical care guidelines for cystic fibrosis-related diabetes: a position statement of the American Diabetes Association and a clinical practice guideline of the Cystic Fibrosis Foundation, endorsed by the Pediatric Endocrine Society. Diabetes Care 2010;33:2697-708.

9. Cystic Fibrosis Foundation. Patient Registry 2002 Annual Data Report. Bethesda, MD: Cystic Fibrosis Foundation, 2003.

10. Cystic Fibrosis Foundation. Patient Registry 2013 Annual Data Report. Bethesda, MD: Cystic Fibrosis Foundation, 2014.

11. James BC. The cystic fibrosis improvement story: we count our successes in lives. BMJ Qual Saf 2014;23:268-71.

12. Hankinson JL, Odencrantz JR, Fedan KB. Spirometric reference values from a sample of the general U.S. population. Am J Respir Crit Care Med 1999;159:179-87.

13. R Development Core Team. R: a language and environment for statistical computing. Vienna, Austria: R Foundation for Statistical Computing, 2010. http://www.R-project.org

14. Zar JH. Biostatistical analysis. 4th edn. Prentice Hall, 1998.

15. Buuren S van Groothuis-Oudshoorn K. MICE: Multivariate Imputation by Chained Equations in R. J Stat Softw 2011;45:1-67.

16. Cystic Fibrosis Foundation. Patient Registry 2004 Annual Data Report. Bethesda, MD: Cystic Fibrosis Foundation, 2004.

17. Dibley MJ, Goldsby JB, Staehling NW, et al. Development of normalized curves for the international growth reference: historical and technical considerations. Am J Clin Nutr 1987;46:736-48.

18. Lai HC, Kosorok MR, Sondel SA, et al. Growth status in children with cystic fibrosis based on the National Cystic Fibrosis Patient Registry data: evaluation of various criteria used to identify malnutrition. J Pediatr 1998;132:478-85. 